\title{
Transcranial magnetic stimulation in cognitive rehabilitation
}

\author{
Carlo Miniussi ${ }^{1,2}$ and Paolo Maria Rossini ${ }^{3,4}$ \\ ${ }^{1}$ Dept of Biomedical Sciences and Biotechnologies, National Institute of \\ Neuroscience, University of Brescia, Brescia, Italy \\ ${ }^{2}$ Cognitive Neuroscience Section, IRCCS San Giovanni di Dio \\ Fatebenefratelli, Brescia, Italy \\ ${ }^{3}$ IRCCS San Raffaele-Pisana and Casa di Cura San Raffaele-Cassino, Italy \\ ${ }^{4}$ Neurology, University Campus Biomedico di Roma, Rome, Italy
}

\begin{abstract}
Repetitive transcranial magnetic stimulation (rTMS) can generate an increase or a decrease of neuronal excitability, which can modulate cognition and behaviour. Transcranial magnetic stimulation-induced cortical changes have been shown to result in neural plasticity. Thus, TMS provides an important opportunity to gain more insight into the mechanisms responsible for the remarkable flexibility of the central nervous system. The aim of this review was to cover the topics that could be useful when using TMS in the cognitive rehabilitation field after brain damage. The basic TMS principles are introduced, together with the clinical application for diagnosis and prognosis, the biological aspects, and the use in cognitive neuroscience studies. Finally, several hypotheses are discussed to explain the likely mechanisms induced by TMS that favour the recovery of a function after brain damage and cause the adult brain to undergo plasticity. The possibility of non-invasively interacting with the functioning of the brain and its plasticity mechanisms - a possibility that may eventually lead to cognitive and behavioural modifications - opens new and exciting scenarios in the cognitive neurorehabilitation field.
\end{abstract}

Keywords: rTMS; Plasticity; Cognition; Neurorehabilitation; Stroke; Noninvasive brain stimulation.

Correspondence should be addressed to Carlo Miniussi, Dept of Biomedical Sciences and Biotechnologies, School of Medicine, University of Brescia, Viale Europa 1125123 Brescia, Italy. E-mail: carlo.miniussi@cognitiveneuroscience.it

The writing of this paper was supported by the James S. McDonnell Foundation (to CM).

(C) 2011 Psychology Press, an imprint of the Taylor \& Francis Group, an Informa business http://www.psypress.com/neurorehab

http://dx.doi.org/10.1080/09602011.2011.562689 


\section{INTRODUCTION}

Recently, non-invasive brain stimulation techniques, such as transcranial magnetic stimulation (TMS), have been developed. These techniques interact with spontaneous brain activity and related sensory-motor and higher order cognitive abilities. Transcranial magnetic stimulation uses a coil to deliver a brief ( $\sim 200$ to $300 \mu \mathrm{s})$ and powerful $(0.2$ to $4.0 \mathrm{~T})$ magnetic pulse to the scalp. The stimulation-induced effects of TMS depend on several technical parameters, including the intensity and number of stimulations (i.e., frequency), the coil orientation, and the focality and depth of stimulation. The effects also depend on a number of variables related to the stimulated subject, including age, eventual pharmacological treatments and the state of the subject (Miniussi, Ruzzoli, \& Walsh, 2010; Rossini, Rossini, \& Ferreri, 2010; Silvanto, Muggleton, \& Walsh, 2008).

When a strong, transient magnetic field is delivered through a coil in close proximity with the scalp, there is a transitory electric current in the cortical surface under the coil, which causes the depolarisation of cell membranes (Barker, Freeston, Jalinous, \& Jarratt, 1987; Barker, Jalinous, \& Freeston, 1985 ) and a transynaptic depolarisation or hyperpolarisation of a population of cortical neurons. Because the electrical field induced by TMS is markedly reduced as the distance from the stimulating coil increases, the cortex $(\sim 2 \mathrm{~cm}$ below the scalp) is the target of this method. TMS originated in the mid1980s, when Barker and colleagues (1985) built the first magnetic stimulator, which was able to excite cortical neurons from the scalp surface. TMS was originally introduced in clinical neurophysiology for the evaluation of the functional state of the corticospinal pathway (Barker et al., 1985, 1987), and TMS initially involved the delivery of single magnetic pulses. When TMS was directed over the primary motor cortex, the discharges induced the activation of the corticospinal tract, which produced a peripheral motor response known as the motor evoked potential (MEP). Since the discovery of TMS, this technique has been used to investigate the state of cortical excitability, the excitability of the cortico-cortical or corticospinal pathway (e.g., Rothwell, Day, Thompson, Dick, \& Marsden, 1987), the role of a given brain region in a particular cognitive function and the timing of its activity (e.g., Walsh \& Pascual-Leone, 2003), and the pathophysiology of various disorders (e.g., Fregni \& Pascual-Leone, 2006; Kobayashi \& Pascual-Leone, 2003; Rossini \& Rossi, 2007).

In the mid-1990s, technological advances allowed the delivery of rhythmic trains of magnetic pulses in a rapid sequence up to a $100-\mathrm{Hz}$ repetition rate, which was referred to as repetitive TMS (rTMS). Studies have shown that rTMS interacts with cortical activity more effectively than TMS. Therefore, new applications began evaluating the potential benefits of rTMS in the treatment of psychiatric disorders (see George et al., 2009 for a review). In recent 
years, rTMS has been rapidly developed as a potential therapeutic tool in many other clinical fields (e.g., Couturier, 2005; Gershon, Dannon, \& Grunhaus, 2003; Hoffman et al., 2005; Hummel \& Cohen, 2006; Mally \& Stone, 2007; Ridding \& Rothwell, 2007; Wassermann \& Lisanby, 2001).

Concerning the use of rTMS, there is a general consensus that low-frequency rTMS consists of continuous trains below $1 \mathrm{~Hz}$, and high-frequency rTMS consists of intermittent trains above $5 \mathrm{~Hz}$ (Rossi et al., 2009). The cut-off between high and low frequencies, however, is not arbitrary. This cut-off is empirically based on direct and indirect measures of brain activity as well as behaviour. Therefore, treating low- and high-frequency rTMS as separate phenomena is essential because the application of these two kinds of stimulation for several minutes might produce distinct effects on brain activity. Converging evidence has indicated that rTMS below $1 \mathrm{~Hz}$ reduces cortical excitability both locally and in functionally related regions, whereas rTMS trains above $5 \mathrm{~Hz}$ seem to have the opposite effect. Namely, high-frequency stimulation has been shown to produce an increase in the MEP amplitude (Maeda, Keenan, Tormos, Topka, \& Pascual-Leone, 2000), whereas low-frequency stimulation produced a decrease in the MEP amplitude (Chen et al., 1997; Maeda et al., 2000). Therefore, rTMS allows for transient modulation of neural excitability, and the effect is dependent on the stimulation frequency and intensity (Thut and Pascal-Leone 2010). Importantly, these effects have been shown to outlast the stimulation period itself. Hebbian mechanisms for synaptic plasticity might be the key for the TMS-induced facilitation. Hebb (1949) postulated that an increase in synaptic efficacy arises when two neurons, the presynaptic and the postsynaptic cells, are activated simultaneously. In this respect, we know that it is possible to induce learning by the repetitive use of a function, which activates specific cortical circuitry. Thus, the same principles may make it possible to induce plasticity by the repetitive use of brain stimulation. Repetitive brain stimulation could generate a prolonged depolarising response and facilitate activity-dependent communications, which would strengthen the circuitry.

Given these premises, there is currently a growing interest in applying TMS as a tentative therapeutic approach in the cognitive rehabilitation field (Rossi \& Rossini 2004). For example, TMS could be used to re-establish cognitive performance in stroke patients (see Miniussi et al., 2008 for a review) and patients suffering from progressive neurodegenerative diseases (Cotelli, Calabria, et al., 2011; Finocchiaro et al., 2006). The aim of this review was to cover the basic principles about TMS that could be useful for cognitive rehabilitation in neurological patients. Interested readers are referred to exhaustive reviews on other TMS-related topics (e.g., George et al., 2009; Hallett, 2000; Rossini \& Rossi, 2007; Sandrini, Umilta, \& Rusconi, 2011; Wassermann et al., 2008). 


\section{BASIC PRINCIPLES}

The primary property of magnetic stimulation is its ability to penetrate all body structures, which allows for the stimulation of regions well below layers of bone (e.g., the brain tissue under the skull). The electric field induced in the tissue generally causes cell membranes to depolarise. If the depolarisation of the membrane overcomes its threshold, an action potential is generated. The locus of activation in the brain appears to be the area where the induced electrical field is maximal (Krings et al., 1997), and this location depends on the stimulating coil characteristics. Focal activation is achieved using a double 70-mm diameter coil with two loops in which the current flows in opposite directions. Also termed the figure-eight coil, it is the one that is most commonly used. With this coil, the induced electric field peaks under the intersection of the coil windings in an area of $\sim 2 \mathrm{~cm}^{2}$ (Thickbroom, Sammut, \& Mastaglia, 1998; Thielscher \& Kammer, 2004). Different coil types are presently available, and each one has its own advantages and disadvantages. For example, large coils cannot produce very focal stimulation. A large volume of neural tissue may be activated by a large coil, but these devices can penetrate relatively deep in the brain (e.g., see the H coil, Roth, Zangen, \& Hallett, 2002; Zangen, Roth, Voller, \& Hallett, 2005). In contrast, small coils produce more focal stimulation but do not penetrate deep into the brain. Indeed, the depth of penetration depends on the coil size, the coil geometry, the intensity of the applied stimulus and individual anatomical factors (e.g., the distance between the cortex and the scalp).

TMS studies on humans were initially focused on the motor system because the effects of TMS on the motor system are easily discernible from peripheral muscles. In the motor cortex, the activation of pyramidal neurons by TMS has been suggested to predominantly occur via interneurons in the superficial cortical layers (Di Lazzaro et al., 2007; Nakamura, Kitagawa, Kawaguchi, \& Tsuji, 1996; Rossini, Caramia, \& Zarola, 1987). Using TMS, studies have shown that it is possible to evaluate the state of motor cortex excitability by measuring what is known as the motor threshold. The motor threshold, which reflects the global excitability of the corticospinal motor pathway, has been defined as the intensity of TMS that produces an identifiable MEP of $\sim 50 \mu \mathrm{V}$ in at least five out of ten TMS pulses (Rossini et al., 1994). Although threshold in human adults is largely independent of age, gender and hemisphere, it varies with different target muscles (Rossini et al., 1987; Rossini, Desiato, \& Caramia, 1992). Differences in the excitability threshold are dependent on the cortical muscle representation (Rothwell et al., 1987). In the upper limbs, for example, the threshold is lowest for the hand muscles and highest for the proximal arm muscles.

Two variables of the MEP that are important in the study of the motor system are amplitude and latency. The amplitude may have a high degree of 
inter-trial and intra-individual variability, especially during stimulating sessions that are only slightly supra-threshold. With increasing stimulus intensity, MEPs increase in amplitude and become less variable in shape. Interestingly, a similar increase in amplitude and decrease in shape variability can be achieved by voluntary (even minimal) contraction of the target muscle. Nevertheless it should be noted that amplitude is never maximal, unless particular techniques of stimulation are used (e.g., triple stimulation techniques, see Rossi et al., 2009). Latency reflects the total motor conduction time from cortex to the target muscle. Latency of the MEP is determined by the fibre diameter, the myelin sheath thickness and the number of synapses that the impulse must cross. There are also several additional factors influencing the amplitude and latency of MEPs. Indeed, a slight voluntary contraction of the target muscle increased excitability and shortened the MEP's onset latency and amplitude (Barker et al., 1987; Di Lazzaro et al., 1998; Rossini et al., 1987; Rothwell et al., 1987). This finding suggested that all of these measures are sensitive to changes in the cortical state, and they can be utilised to evaluate changes (increases or decreases) in the excitability of the motor cortex that are induced by different types of protocols. Therefore, TMS can be used on the motor cortex of conscious human subjects as a non-invasive technique to study neuroplasticity. Several paradigms have been developed, and TMS can be applied one stimulus at a time (single-pulse TMS), in pairs of stimuli separated by a variable and programmable interval (paired-pulse TMS), or in patterned stimulation (for a precise classification see Rossi et al., 2009).

Single-pulse TMS applied on the scalp overlying the primary motor cortex (M1) allows for routine evaluations of the excitability and conductivity of corticospinal motor pathways. This approach has primarily been applied in studies of movement physiology in healthy subjects, in patients with neurological disorders and in post-lesion follow-up studies of plastic cerebral reorganisation.

Under certain technical circumstances, paired-pulse techniques have been shown to provide measures of intracortical facilitation and inhibition as well as cortico-cortical interactions, which are important when evaluating changes in system state or functionality (Nakamura, Kitagawa, Kawaguchi, \& Tsuji, 1997; Sommer, Tergau, Wischer, \& Paulus, 2001). Moreover, plastic modifications of intracortical excitability can be achieved by pairing a peripheral nerve stimulus to a single TMS stimulus of the motor cortex (i.e., paired associative stimulation - PAS; Classen et al., 2004; Mariorenzi, Zarola, Caramia, Paradiso, \& Rossini, 1991; Stefan, Kunesch, Benecke, Cohen, \& Classen, 2002).

When multiple stimuli of TMS are delivered in trains, one can differentiate conventional and patterned protocols of repetitive stimulation. For conventional protocols, there is agreement on the term rTMS. When the motor system is targeted with a repetitive rhythmic stimulation for several 
minutes, rTMS effects have been fairly consistent and appear to cause longlasting neurophysiological effects. As previously reported, continuous lowfrequency rTMS above threshold reduced cortical excitability, which was measured by a decrease in MEP amplitude. Sequences of intermittent high frequency, however, appeared to facilitate cortical excitability and induce an increase in MEP amplitude (Fitzgerald, Fountain, \& Daskalakis, 2006).

Patterned rTMS refers to a repetitive application of short rTMS bursts at a high inner frequency, which are separated by short pauses of no stimulation. To date, theta burst stimulation (TBS) is the most commonly used method of patterned rTMS. In TBS, short bursts of 50-Hz rTMS are repeated as a continuous or intermittent train at a rate in the theta range $(5 \mathrm{~Hz})$. The excitatory and inhibitory effects of this type of stimulation can be manipulated by continuous or intermittent delivery of these theta bursts over time (Huang, Edwards, Rounis, Bhatia, \& Rothwell, 2005). This experimental approach demonstrated that a short, low-intensity TBS protocol transiently modified the excitability of the motor cortex, which outlasted the period of actual TMS for up to one hour. The TBS protocol has now been used to modulate motor thresholds (Huang et al., 2005), visual cortex excitability (Silvanto, Muggleton, Cowey, \& Walsh, 2007 even if they used a modified protocol, i.e., 8 pulses at $40 \mathrm{~Hz}$, separated by $1800 \mathrm{~ms}$ for $50 \mathrm{sec}$ ), and cognitive functions (Vallesi, Shallice, \& Walsh, 2007; Waterston \& Pack, 2010). Recently, quadripulse stimulation (QPS), which is able to induce long-term changes in cortical excitability, has been added to the patterned rTMS procedures (Hamada et al., 2008). In addition, QPS might also be used to induce symptomatic relief of several types of neurological symptoms (Hamada \& Ugawa, 2010).

\section{CLINICAL APPLICATION OF TMS FOR DIAGNOSIS AND PROGNOSIS IN THE MOTOR SYSTEM}

The high reproducibility in standardised conditions makes MEPs efficacious for evaluating corticospinal tract functionality, even in a sub-clinical involvement of the central motor pathways (Rossini \& Rossi, 1998). Characteristics of MEPs rely on the number of neurons recruited and the integrity of the direct corticospinal tract. Thus, in patients with spinal cord disorders, MEPs may be useful in diagnostic procedures to demonstrate the site of spinal cord lesion and monitor the disease progression. The ability of TMS to predict the quality of motor recovery in stroke patients has yielded contradictory results, which were likely caused by variability in the clinical pictures of recruited patients. Most authors agree, however, that the evoked potentials measured in the acute stage have predictive value (Binkofski et al., 1996; Cicinelli, Traversa, \& Rossini, 1997). For instance, the absence of a response to TMS in the first 48 hours after a stroke has been shown to be predictive of 
an absent or a very poor functional hand motor recovery (Binkofski et al., 1996; Cicinelli et al., 1997; Nardone \& Tezzon, 2002; Pennisi et al., 1999). Follow-up of stroke patients undergoing neurorehabilitation of the upper limb showed an increase in the number of cortical sites where an MEP of the paretic hand could be elicited, which suggested a cortical reorganisation of the area representing the hand (Liepert et al., 1998, 2000; Miltner, Bauder, Sommer, Dettmers, \& Taub, 1999; Traversa, Cicinelli, Bassi, Rossini, \& Bernardi, 1997; Wittenberg et al., 2003). Therefore, TMS may provide fruitful information regarding the final functional outcome on the motor system (see Rossini, Calautti, Pauri, \& Baron, 2003).

Presently, a peripheral "functional" marker (i.e., MEP) is present over the motor cortex, which can be used to directly measure the state of the cortex. In addition, other induced responses can also be recorded (e.g., the primary visual cortices can be localised for inducing the perception of contralateral visual phosphenes, and a visual phosphene induction threshold can be set) (Kammer \& Baumann, 2010). The combination of TMS with simultaneous electroencephalography (EEG) might allow direct measurements of the state of all cortical areas. Similar to the way that MEPs recorded from muscles after TMS over motor cortex are markers of the state of the motor system, TMS-evoked potentials recorded over the scalp might be quantifiable markers of the cerebral neurophysiological state in "silent areas". Therefore, an integration of TMS and EEG might provide real-time information on the excitability of the cortex as well as the link between functional activity and behaviour modifications (Komssi \& Kahkonen, 2006; Miniussi \& Thut, 2010). TMS EEG coregistration has only recently become feasible because of technical developments (Ilmoniemi et al., 1997; Virtanen, Ruohonen, Naatanen, \& Ilmoniemi, 1999), and a great deal of work remains to be done.

\section{BIOLOGICAL ASPECTS}

Studies have shown that $1-\mathrm{Hz}$ cortical inhibitory effects on a stimulated area were dependent on both GABA and NMDA receptor system activity (Fitzgerald et al., 2005), whereas high-frequency stimulation might rely on the same system but have opposite effects. Moreover, these effects can be altered by drugs that specifically interact with neurotransmission in the GABA and NMDA receptor systems (Ziemann, Chen, Cohen, \& Hallett, 1998; Ziemann, Hallett, \& Cohen, 1998). Therefore, both long-term depression (LTD) and long-term potentiation (LTP) have been postulated as likely mechanisms to explain the persistent effects of rTMS on cortical activity (Cooke \& Bliss, 2006; Thickbroom, 2007). For instance, one study showed that rTMS (from 1 to $10 \mathrm{~Hz}$ ) to the auditory cortex of gerbils resulted in a frequency-dependent LTD-like suppression or LTP-like enhancement of 
auditory cortex activity congruent with the stimulation frequency (Wang, Wang, \& Scheich, 1996). Recently, studies have proposed that genetic factors might also influence the response to TMS plasticity protocols, and particular attention has been paid to the brain-derived neurotrophic factor (BDNF) gene. The mature form of BDNF is a neurotrophin that plays a pivotal role in neurodevelopment because it regulates and promotes synaptic plasticity mechanisms (Egan et al., 2003). Single-pulse TMS has been used to demonstrate that the BDNF genotype was associated with changes in the excitability of the primary motor cortex, which occurred after practising a motor task (Kleim et al., 2006). In addition, the response of healthy subjects to three different plasticity-inducing protocols in the motor cortex was associated with the polymorphism of the BDNF gene that they carried (Cheeran et al., 2008). In addition, experiments in rats showed that the administration of BDNF after stroke improved recovery (Schabitz et al., 2004). Interestingly, the physiological consequences of the BDNF polymorphism might not be manifested in the basal state, but they may become evident in response to a behaviourally driven increase in neural activity. Thus, genetic variations in patients could produce significant differences in the brain stimulation protocols (Bocchio-Chiavetto et al., 2008).

\section{TMS IN THE FIELD OF COGNITIVE NEUROSCIENCE}

The use of TMS in the field of cognitive neuroscience depends mainly on its ability to transiently interact with the stimulated neural network rather than its ability to modulate cortical excitability. Therefore, rTMS can be used with two distinct approaches: on-line stimulation and off-line stimulation. Interaction with on-going cognitive processing when TMS or rTMS is applied during the performance of a task is called on-line TMS (Sandrini et al., 2011). In this case, it is generally assumed that an alteration of cortical activity within a specific targeted area can significantly impair performance (Walsh \& Pascual-Leone, 2003; but see Miniussi et al., 2010). Moreover, the effects induced by on-line stimulation are generally short-lived, probably on the order of a few hundred milliseconds to a few seconds. Therefore, the idea that increasing excitability via high-frequency stimulation will lead to a behaviourally measurable modification is barely applicable. Both facilitation and inhibition, which are timing, site and task specific, have been reported irrespective of the frequency of stimulation (Boroojerdi et al., 2001; Cappa, Sandrini, Rossini, Sosta, \& Miniussi, 2002; Harris, Benito, Ruzzoli, \& Miniussi, 2008; Kirschen, Davis-Ratner, Jerde, Schraedley-Desmond, \& Desmond, 2006; Kohler, Paus, Buckner, \& Milner, 2004; Luber et al., 2007; Mottaghy et al., 1999; Sparing et al., 2001).

In the case of off-line stimulation, rTMS is applied for several minutes before the subject is tested on a task. In this case, rTMS affects the modulation 
of cortical excitability (increase vs. decrease) and aims to change the cognitive performance. Studies, however, have not always confirmed the idea of a strict and unequivocal association between both behavioural improvements and excitation and disruptions and inhibition (Andoh et al., 2006; Drager, Breitenstein, Helmke, Kamping, \& Knecht, 2004; Hilgetag, Theoret, \& Pascual-Leone, 2001; Kim et al., 2005; Waterston \& Pack, 2010). Nevertheless, modification of the activity of a neural network by rTMS carries important behavioural implications for neurorehabilitation, which will be considered later.

A lot of evidence has suggested that the effects induced by several off-line rTMS approaches were site specific, but not site limited (Bestmann et al., 2008). Thus, the long-term consequences induced by sustained repetitive brain stimulation were most likely due to activity changes in a given network of cortical and subcortical areas rather than a local inhibition or excitation of an individual brain area (Selimbeyoglu \& Parvizi, 2010). In other words, brain stimulation can modulate the ongoing properties of a neuronal network by amplification or reduction of its activity. Because the brain operates through flexible and interactive distributed networks (primarily for cognition and behaviour), we can expect that the modification of a node of the network would affect the entire network. Moreover, the stimulated area cannot be considered to be isolated from its own functions or the functional status induced by the state of the subject (Harris et al., 2008; Pasley, Allen, \& Freeman, 2009; Ruzzoli, Marzi, \& Miniussi, 2010; Silvanto et al., 2008). These aspects suggest that the functional effects induced in one area could be co-opted into different functions depending on the mode of activation or which of its interconnected networks was activated (e.g., Harris, Clifford, \& Miniussi, 2008; Selimbeyoglu \& Parvizi, 2010; Silvanto, Cowey, Lavie, \& Walsh, 2005).

In general, two of the great advantages of TMS are that it can be used in a large group of subjects, and the location of the coil can be precisely controlled with the neuronavigation approach. The manipulation with rTMS can be meaningful if the coil positioning can be accurately localised on an individual basis, particularly in those functions where interindividual differences are particularly relevant (Manenti et al., 2010). Therefore in some cases it is very important to guide the positioning of the coil over the target area by neuronavigation systems with single subject functional magnetic resonance imaging (Sack et al., 2009).

Another advantage is that TMS can be applied at different time points during the execution of a cognitive task. Thus, TMS can provide valuable information about when a brain region is involved in a cognitive task. Ultimately, TMS could be used to map the flow of information across different brain regions during the execution of a complex cognitive task.

Occasionally, some technical aspects of rTMS (e.g., its spatial resolution, the noise produced and the possibility of a general arousal induction) have 
hindered precise interpretation of the observed functional effects. Thus, a control condition must be used to strengthen the results of rTMS experiments. Several approaches could be used to ensure that changes in performance are specifically attributed to the effects of TMS on the target site. One common approach has been the use of sham (i.e., placebo) stimulation as a baseline condition. Although all parameters were the same as actual stimulation, no effective magnetic stimulation should reach the brain in the sham condition (Rossi et al., 2007; please note that tilting the coil by $180^{\circ}$ is not a sham condition). Another approach involves stimulating contralateral homologous (i.e., homotopic) or vertex areas while the subject performs the same task with auditory and somatosensory perceptions being the same. This allows for a comparison of rTMS effects at different brain sites, but only one has functional relevance. In addition, subject behaviour can also be observed across several distinct tasks. Following stimulation at one site, however, only one task is functionally related to the stimulated site.

All of these technical/control aspects are very important in experiments that involve cognition because the functional effects that can be induced after stimulation of a cortical area can take on different functions depending on which of its interconnected networks is engaged in a given task (Sack \& Linden, 2003). In addition, there are ethical implications of TMS application because attempting to modify "natural" cerebral abilities raises concerns, which share some similarities with the problems connected with neurodoping.

\section{TMS AND COGNITIVE NEUROREHABILITATION}

Several studies in normal subjects have suggested that TMS may lead to enhanced cognitive performance (Vallar \& Bolognini, this issue). In healthy subjects, most of these effects were transient (in the range of minutes), but repeated TMS in follow-up sessions, in concert with learning and plasticity processes, may prolong the facilitating effects beyond the end of the stimulation period and provide important opportunities for longlasting positive effects (Miniussi et al., 2008). Some preliminary data have shown improved picture naming or word repetition performance in vascular aphasia (Cotelli, Fertonani, et al., this issue; Kakuda, Abo, Kaito, Watanabe, \& Senoo, 2010; Martin et al., 2004; Naeser et al., 2010; Naeser, Martin, Nicholas, Baker, Seekins, Helm-Estabrooks et al., 2005; Naeser, Martin, Nicholas, Baker, Seekins, Kobayashi et al., 2005), primary progressive aphasia (Finocchiaro et al., 2006) and Alzheimer's disease (Cotelli, Calabria, et al., 2011). Repetitive TMS can also be used to improve performance in sensory extinction (Oliveri et al., 1999, 2000) and unilateral neglect (Brighina et al., 2003; Shindo et al., 2006) associated with stroke. 
The general idea behind brain stimulation is that inducing changes in cortical excitability leads to a recovery or reorganisation of the functional network responsible for the impaired cognitive function. Function may be restored or compensated by mechanisms that involve both structural and functional changes of the brain circuits. In addition, the restored function might be based on the same general rules that were valid during the development of the nervous system or during learning-dependent plasticity. Even if adaptation of the adult brain relies on functional plasticity rather than growth or maturation, there are several potential mechanisms to explain restored functioning. Indeed, there could be a gradual readjustment of an intact but functionally suppressed area (initially due to diaschisis but then due to a steady reduction in synaptic strength). Following a lesion, a reduction of the excitability of cortical neurons within the affected area might induce a depression/depotentiation of the cognitive circuit underlying the function, which would result in an impaired function carried on by a "non-specialised area". Recovery of a function might be due to:

1. An enhancement of cortical excitability by focal brain stimulation of a given network might change this maladaptive pattern through a specific potentiation-like phenomenon, which would enable synaptic plasticity and promote recovery of the degraded function. A similar mechanism might work in Alzheimer's patients because neuronal death is not an all-or-none phenomenon. Even in severely affected areas, studies have shown evidence of plastic changes in surviving neurons (Backman et al., 1999; Becker et al., 1996). In Alzheimer's patients, it has been shown that rTMS can induce a partial recovery of language abilities (Cotelli, Calabria, et al., 2011; Finocchiaro et al., 2006), which may be due to a strengthening of the synaptic activity of the surviving neurons in the stimulated network. Following the loss of a part of the neural population, a reduction of excitability of cortical neurons within the affected area might induce a depression/depotentiation of the cognitive circuit underling the function, resulting in an impaired function. The same phenomena can be present in stroke patients. After a lesion of an area adjacent or connected, areas become "silent" due to diaschisis, therefore lesion-induced effects are a weakening of the synaptic activity resulting in silent synapses. TMS might induce a gradual readjustment of an intact but "functionally" suppressed area due to a steady reduction in synaptic strength. Therefore, these data supported the idea that brain stimulation-induced changes in synaptic strength are an essential step towards recovery of function. Indeed, improving the performance of a specific system within the functional network leads to more effective processing.

2. Recruitment or rebalance of compensatory networks may be used to accomplish the impaired function. These networks may include 
perilesional areas and/or contralateral homologue cortical regions, which might have similar anatomical structure and can therefore carry out the impaired functions. This type of compensatory function has been promoted by specific cognitive rehabilitative interventions, such as brain stimulation, which aim to exploit the preserved abilities to compensate for the deficit.

3. Early intervention might even favour the restoration of neural function by regeneration of damaged neurons or partially lost connections. We know that BDNF promotes synaptic plasticity mechanisms and is essential for neuronal cell survival and differentiation. Indeed, studies in rats with a cerebral infarct have shown that BDNF was significantly increased after rTMS treatment, which suggested that TMS was able to promote BDNF expression in the cerebral cortex (Zhang, Mei, Liu, \& Yu, 2007) and promote neuronal cell survival.

4. In addition, a number of investigations have also suggested that the rhythmic aspects of transcranial stimulation can exert positive effects on cognitive performance. Interestingly, the modification of cortical oscillatory activity through the use of rhythmic repetitive stimulation (Barr et al., 2009; Klimesch, Sauseng, \& Gerloff, 2003) may readjust pathological patterns of brain activity. This would allow an opportunity to induce new oscillatory patterns, which could adequately modulate the neural response of a network (i.e., entrainment) (Thut \& Miniussi, 2009).

All of these mechanisms are likely to be the same ones that favour the naturally occurring recovery of a function after brain damage and cause the adult brain to undergo plasticity. Interestingly, lesion-induced plasticity has been suggested to be stronger when it occurs shortly after the occurrence of the damaging event (Clarkson \& Carmichael, 2009), and this plasticity becomes weaker as more time passes (Carmichael, 2006). Therefore, brain stimulation might create more time for plasticity to occur, which would favour functional compensation.

In behavioural paradigms, inducing plasticity when a stimulus is cognitively associated with reinforcement allows its cortical representation to be strengthened and enlarged (Blake, Heiser, Caywood, \& Merzenich, 2006). Several experiments have shown that plasticity changes were not caused by sensory experience alone; they also require learning and reward to be associated with the sensory experience (Blake et al., 2006). This finding suggested that a simple stimulation of the brain by TMS, which was not associated with a focused cognitive rehabilitation procedure that could be fully experienced by the patient, might prove to induce weak and transient effects. Moreover, BDNF synaptic modulation is also activity-dependent, and therefore activity could control the synapse specificity of neurotrophin regulation-supported recovery (Lu, 2003). 
All in all, these considerations advocate that TMS leads to the stimulationinduced modulation of network activity rather than simple excitation or inhibition of the target area (McIntyre, Savasta, Walter, \& Vitek, 2004). Therefore, TMS could be used to achieve a strengthening or modification of a network that is specific for a diminished cognitive function. Studies have suggested that the best way to perpetrate this strengthening is to stimulate the area and activate the network supporting the specific function (Barbay et al., 2006). This approach can be achieved by combining exogenously induced plasticity (i.e., brain stimulation) with a specific training-induced plasticity (i.e., cognitive training). In part, this approach resembles the one used to improve motor performance in patients with hemiplegia (for discussion on this issue see Fregni \& Pascual-Leone, 2007; Hummel \& Cohen, 2006; Nowak, Grefkes, Ameli, \& Fink, 2009; Talelli \& Rothwell, 2006). If brain stimulation is applied when the system is in a given functional state, it will enhance and strengthen the specific distributed functional cortico-cortical (or subcortical) network that is active rather than inducing a non-specific arousal or activation of the system.

Many additional aspects need to be considered in future studies, such as the timing of rTMS application after stroke, the duration of the rehabilitation protocol, the type of frequency and the ideal area that should be stimulated. For example, studies have not determined if the facilitation of the affected area (Meinzer et al., 2008; but see also Kleim et al., 2003; Nudo, Wise, SiFuentes, $\&$ Milliken, 1996; Plautz et al., 2003) or the inhibition of the unaffected contralateral hemisphere (Kakuda et al., 2010; Martin et al., 2004) is more effective in improving the hampered function (see the interhemispheric competition model, Ward \& Cohen, 2004). In addition, some researchers studying the motor cortex have suggested that the stimulation of both areas would be the most effective strategy (Takeuchi, Tada, Toshima, Matsuo, \& Ikoma, 2009). Because motor cortex is a primary area, it has a similar functional representation on both hemispheres. Cognitive functions that do not rely on primary areas and are unilaterally localised, however, might not follow the same logic. In addition, the parameters that induce a clear result in a normal system may lead to an opposite pattern of results in a pathological system that has a different homeostasis (for a review on variability in inducing cortical plasticity in normal subjects see Ridding \& Ziemann, 2010). Furthermore, cortical stimulation in future applications might include combined rTMS and transcranial electrical stimulation (see Paulus, this issue) to induce modifications of cortical responsiveness.

\section{SAFETY}

According to recently published safety guidelines, the risk of TMS use should be assessed carefully, and its dosage should generally be limited (Rossi et al., 
2009). Since the introduction of TMS, many thousands of subjects have been examined with TMS or rTMS to assess motor or cognitive functions of the central nervous system. There is now a considerable volume of data supporting the safety of magnetic stimulation. The main area of concern has been the triggering of kindling activity, which can induce seizures. Of course, stroke is a factor that may increase the possibility of inducing seizures since it affects cortical excitability. Therefore, rTMS trains that are usually safe for healthy volunteers might potentially increase the risk of seizures in these patients. In stroke patients the current density distributions can be modified in magnitude and direction by the altered structure, potentially altering the population of stimulated neural elements (Rossi et al., 2009). This needs to be considered when assessing the safety of rTMS treatment and rigorous monitoring is critical. In addition, patients will likely be taking medications, and interactions between rTMS and concomitant treatments should be considered (Rossi et al., 2009) because several neuroleptics and antidepressants also increase seizure risk. Seizure induction, however, has rarely been reported following rTMS, and animal studies have shown that there is no clear evidence that rTMS leads to increased seizure susceptibility (Akamatsu et al., 2001; Ebert \& Ziemann, 1999; Fleischmann, Hirschmann, Dolberg, Dannon, \& Grunhaus, 1999; Fleischmann, Prolov, Abarbanel, \& Belmaker, 1995; Godlevsky et al., 2006; Post, Muller, Engelmann, \& Keck, 1999), structural alterations (Counter, Borg, \& Olofsson, 1993; de Sauvage, Lagroye, Billaudel, \& Veyret, 2008; Liebetanz, Nitsche, \& Paulus, 2003; Post et al., 1999; Sgro, Ghatak, Stanton, Emerson, \& Blair, 1991) or off-line impairment of cognitive functions (Post et al., 1999), even after long-term treatments. Researchers who are interested in using TMS are indeed encouraged to read the comprehensive document that has recently been created by a large panel of international experts (Rossi et al., 2009).

\section{CONCLUSIONS}

Further investigations are needed to determine whether TMS can be used to ameliorate deficits in the cognitive domain with long-lasting effects. An important aspect is that TMS cannot be considered the treatment by itself but it should give us the opportunity, when the associated with a focused cognitive rehabilitation, to reduce the treatment time and potentiate the final effects. Indeed, it will represent a challenging field of research for years to come. There are several aspects that should be considered to control all of the variables that could hamper a neurorehabilitation protocol. Protocol that should consider the full involvement and motivation by the patient. Perhaps the most important consideration should be that the goal of the treatment may not be to restore the function of damaged components. Indeed, the 
goal could be exploitation of the preserved abilities to compensate for the deficit and/or to speed-up progressive ("plastic") recruitment of new neural networks, which might re-establish lost or degraded functions. Therefore, it is important to determine precisely the anatomo-functional state of each individual subject and establish the location and extension of the damaged area, its neurophysiological state (e.g., by means of TMS EEG co-registration methods) and the state of the perilesional cortex. Because the mechanisms of recovery from stroke could be strictly related to the type of damage, the potential cognitive strategy to be used in combination with brain stimulation could be chosen based on the preserved regions. In addition, brain stimulation is applied to an ongoing brain activity, which is determined by both lesioninduced plasticity mechanisms and new behavioural learning mechanisms. All of these matters should be evaluated in the context of the internal milieu of the central nervous system, which will try to maintain a balance (likely through homeostatic plasticity). In terms of maintaining balance in the CNS, there may also be negative plasticity that contributes to the deficits and/or symptoms interfering with normal functions (e.g., spasticity or abnormal reflexes). Thus, the goal of rehabilitative interventions should be to try to favour positive neuroplastic changes. Natural recovery mechanisms must also be considered as important components of stroke rehabilitation studies because natural effects can also contribute to enhanced physical, psychological and social functioning. In the search for more effective and long-lasting effects of neurorehabilitation, spontaneous recovery should be included in neurorehabilitation studies as an important variable that can be eventually potentiated by brain stimulation. In addition, spontaneous recovery in search of a new balance might induce maladaptive neural plasticity that could be avoided with the adequate guidance of a well-designed rehabilitation protocol.

\section{REFERENCES}

Akamatsu, N., Fueta, Y., Endo, Y., Matsunaga, K., Uozumi, T., \& Tsuji, S. (2001). Decreased susceptibility to pentylenetetrazol-induced seizures after low-frequency transcranial magnetic stimulation in rats. Neuroscience Letters, 310(2-3), 153-156.

Andoh, J., Artiges, E., Pallier, C., Riviere, D., Mangin, J. F., Cachia, A., et al. (2006). Modulation of language areas with functional MR image-guided magnetic stimulation. Neuroimage, 29(2), 619-627.

Backman, L., Andersson, J. L., Nyberg, L., Winblad, B., Nordberg, A., \& Almkvist, O. (1999). Brain regions associated with episodic retrieval in normal aging and Alzheimer's disease. Neurology, 52(9), 1861-1870.

Barbay, S., Plautz, E. J., Friel, K. M., Frost, S. B., Dancause, N., Stowe, A. M., et al. (2006). Behavioral and neurophysiological effects of delayed training following a small ischemic infarct in primary motor cortex of squirrel monkeys. Experimental Brain Research, 169(1), 106-116. 
Barker, A. T., Freeston, I. L., Jalinous, R., \& Jarratt, J. A. (1987). Magnetic stimulation of the human brain and peripheral nervous system: An introduction and the results of an initial clinical evaluation. Neurosurgery, 20(1), 100-109.

Barker, A. T., Jalinous, R., \& Freeston, I. L. (1985). Non-invasive magnetic stimulation of human motor cortex. Lancet, 1(8437), 1106-1107.

Barr, M. S., Farzan, F., Rusjan, P. M., Chen, R., Fitzgerald, P. B., \& Daskalakis, Z. J. (2009). Potentiation of gamma oscillatory activity through repetitive transcranial magnetic stimulation of the dorsolateral prefrontal cortex. Neuropsychopharmacology, 34(11), 2359-2367.

Becker, J. T., Mintun, M. A., Aleva, K., Wiseman, M. B., Nichols, T., \& DeKosky, S. T. (1996). Compensatory reallocation of brain resources supporting verbal episodic memory in Alzheimer's disease. Neurology, 46(3), 692-700.

Bestmann, S., Ruff, C. C., Blankenburg, F., Weiskopf, N., Driver, J., \& Rothwell, J. C. (2008). Mapping causal interregional influences with concurrent TMS-fMRI. Experimental Brain Research, 191(4), 383-402.

Binkofski, F., Seitz, R. J., Arnold, S., Classen, J., Benecke, R., \& Freund, H. J. (1996). Thalamic metabolism and corticospinal tract integrity determine motor recovery in stroke. Annals of Neurology, 39(4), 460-470.

Blake, D. T., Heiser, M. A., Caywood, M., \& Merzenich, M. M. (2006). Experience-dependent adult cortical plasticity requires cognitive association between sensation and reward. Neuron, 52(2), 371-381.

Bocchio-Chiavetto, L., Miniussi, C., Zanardini, R., Gazzoli, A., Bignotti, S., Specchia, C., et al. (2008). 5-HTTLPR and BDNF Val66Met polymorphisms and response to rTMS treatment in drug resistant depression. Neuroscience Letters, 437(2), 130-134.

Boroojerdi, B., Phipps, M., Kopylev, L., Wharton, C. M., Cohen, L. G., \& Grafman, J. (2001). Enhancing analogic reasoning with rTMS over the left prefrontal cortex. Neurology, 56(4), 526-528.

Brighina, F., Bisiach, E., Oliveri, M., Piazza, A., La Bua, V., Daniele, O., et al. (2003). 1 Hz repetitive transcranial magnetic stimulation of the unaffected hemisphere ameliorates contralesional visuospatial neglect in humans. Neuroscience Letters, 336(2), 131-133.

Cappa, S. F., Sandrini, M., Rossini, P. M., Sosta, K., \& Miniussi, C. (2002). The role of the left frontal lobe in action naming: rTMS evidence. Neurology, 59(5), 720-723.

Carmichael, S. T. (2006). Cellular and molecular mechanisms of neural repair after stroke: Making waves. Annals of Neurology, 59(5), 735-742.

Cheeran, B., Talelli, P., Mori, F., Koch, G., Suppa, A., Edwards, M., et al. (2008). A common polymorphism in the brain-derived neurotrophic factor gene (BDNF) modulates human cortical plasticity and the response to rTMS. Journal of Physiology, 586(Pt 23), 5717-5725.

Chen, R., Classen, J., Gerloff, C., Celnik, P., Wassermann, E. M., Ha, M., et al. (1997). Depression of motor cortex excitability by low-frequency transcranial magnetic stimulation. Neurology, 48(5), 1398-1403.

Cicinelli, P., Traversa, R., \& Rossini, P. M. (1997). Post-stroke reorganization of brain motor output to the hand: A 2-4 month follow-up with focal magnetic transcranial stimulation. Electroencephalography and Clinical Neurophysiology, 105(6), 438-450.

Clarkson, A. N., \& Carmichael, S. T. (2009). Cortical excitability and post-stroke recovery. Biochemical Society Transactions, 37(Pt 6), 1412-1414.

Classen, J., Wolters, A., Stefan, K., Wycislo, M., Sandbrink, F., Schmidt, A., et al. (2004). Paired associative stimulation. Supplemental Clinical Neurophysiology, 57, 563-569.

Cooke, S. F., \& Bliss, T. V. (2006). Plasticity in the human central nervous system. Brain, 129(Pt 7), 1659-1673.

Cotelli, M., Calabria, M., Manenti, R., Rosini, S., Zanetti, O., Cappa, S. F., et al. (2011). Improved language performance in Alzheimer disease following brain stimulation. 
Journal of Neurology Neurosurgery and Psychiatry. Advance online publication. doi:10.1136/jnnp.2009.197848

Cotelli, M., Fertonani, A., Miozzo, A., Rosini, S., Manenti, R., Padovani, A., et al. (2011). Anomia training and brain stimulation in chronic aphasia. Neuropsychological Rehabilitation, 21(5), 717-741.

Counter, S. A., Borg, E., \& Olofsson, A. (1993). Oto-traumatic effects of computer simulated magnetic coil impulse noise: Analysis of mechanisms. Acta Otolaryngology, 113(6), 699-705.

Couturier, J. L. (2005). Efficacy of rapid-rate repetitive transcranial magnetic stimulation in the treatment of depression: A systematic review and meta-analysis. Journal of Psychiatry Neuroscience, 30(2), 83-90.

de Sauvage, R. C., Lagroye, I., Billaudel, B., \& Veyret, B. (2008). Evaluation of the potential genotoxic effects of rTMS on the rat brain and current density mapping. Clinical Neurophysiology, 119(2), 482-491.

Di Lazzaro, V., Restuccia, D., Oliviero, A., Profice, P., Ferrara, L., Insola, A., et al. (1998). Effects of voluntary contraction on descending volleys evoked by transcranial stimulation in conscious humans. Journal of Physiology, 508(Pt 2), 625-633.

Di Lazzaro, V., Thickbroom, G. W., Pilato, F., Profice, P., Dileone, M., Mazzone, P., et al. (2007). Direct demonstration of the effects of repetitive paired-pulse transcranial magnetic stimulation at I-wave periodicity. Clinical Neurophysiology, 118(6), 1193-1197.

Drager, B., Breitenstein, C., Helmke, U., Kamping, S., \& Knecht, S. (2004). Specific and nonspecific effects of transcranial magnetic stimulation on picture-word verification. European Journal of Neuroscience, 20(6), 1681-1687.

Ebert, U., \& Ziemann, U. (1999). Altered seizure susceptibility after high-frequency transcranial magnetic stimulation in rats. Neuroscience Letters, 273(3), 155-158.

Egan, M. F., Kojima, M., Callicott, J. H., Goldberg, T. E., Kolachana, B. S., Bertolino, A., et al. (2003). The BDNF val66met polymorphism affects activity-dependent secretion of BDNF and human memory and hippocampal function. Cell, 112(2), 257-269.

Finocchiaro, C., Maimone, M., Brighina, F., Piccoli, T., Giglia, G., \& Fierro, B. (2006). A case study of Primary Progressive Aphasia: Improvement on verbs after rTMS treatment. Neurocase, 12(6), 317-321.

Fitzgerald, P. B., Benitez, J., Oxley, T., Daskalakis, J. Z., de Castella, A. R., \& Kulkarni, J. (2005). A study of the effects of lorazepam and dextromethorphan on the response to cortical $1 \mathrm{~Hz}$ repetitive transcranial magnetic stimulation. Neuroreport, 16(13), 1525-1528.

Fitzgerald, P. B., Fountain, S., \& Daskalakis, Z. J. (2006). A comprehensive review of the effects of rTMS on motor cortical excitability and inhibition. Clinical Neurophysiology, 117(12), 2584-2596.

Fleischmann, A., Hirschmann, S., Dolberg, O. T., Dannon, P. N., \& Grunhaus, L. (1999). Chronic treatment with repetitive transcranial magnetic stimulation inhibits seizure induction by electroconvulsive shock in rats. Biological Psychiatry, 45(6), 759-763.

Fleischmann, A., Prolov, K., Abarbanel, J., \& Belmaker, R. H. (1995). The effect of transcranial magnetic stimulation of rat brain on behavioral models of depression. Brain Research, 699(1), 130-132.

Fregni, F., \& Pascual-Leone, A. (2006). Hand motor recovery after stroke: Tuning the orchestra to improve hand motor function. Cognitive Behavioural Neurology, 19(1), 21-33.

Fregni, F., \& Pascual-Leone, A. (2007). Technology insight: non-invasive brain stimulation in neurology-perspectives on the therapeutic potential of rTMS and tDCS. Nature Clinical Practice Neurology, 3(7), 383-393.

George, M. S., Padberg, F., Schlaepfer, T. E., O'Reardon, J. P., Fitzgerald, P. B., Nahas, Z. H., et al. (2009). Controversy: Repetitive transcranial magnetic stimulation or transcranial direct current stimulation shows efficacy in treating psychiatric diseases (depression, 
mania, schizophrenia, obsessive-compulsive disorder, panic, posttraumatic stress disorder). Brain Stimulation, 2(1), 14-21.

Gershon, A. A., Dannon, P. N., \& Grunhaus, L. (2003). Transcranial magnetic stimulation in the treatment of depression. American Journal of Psychiatry, 160(5), 835-845.

Godlevsky, L. S., Kobolev, E. V., van Luijtelaar, E. L., Coenen, A. M., Stepanenko, K. I., \& Smirnov, I. V. (2006). Influence of transcranial magnetic stimulation on spike-wave discharges in a genetic model of absence epilepsy. Indian Journal of Experimental Biology, 44(12), 949-954.

Hallett, M. (2000). Transcranial magnetic stimulation and the human brain. Nature, 406(6792), $147-150$.

Hamada, M., Terao, Y., Hanajima, R., Shirota, Y., Nakatani-Enomoto, S., Furubayashi, T., et al. (2008). Bidirectional long-term motor cortical plasticity and metaplasticity induced by quadripulse transcranial magnetic stimulation. Journal of Physiology, 586(16), 3927-3947.

Hamada, M., \& Ugawa, Y. (2010). Quadripulse stimulation: A new patterned rTMS. Restor Neurology Neuroscience, 28(4), 419-424.

Harris, I. M., Benito, C. T., Ruzzoli, M., \& Miniussi, C. (2008). Effects of right parietal transcranial magnetic stimulation on object identification and orientation judgments. Journal of Cognitive Neuroscience, 20(5), 916-926.

Harris, J. A., Clifford, C. W., \& Miniussi, C. (2008). The functional effect of transcranial magnetic stimulation: Signal suppression or neural noise generation? Journal of Cognitive Neuroscience, 20(4), 734-740.

Hebb, D. O. (1949). The Organization of Behavior: A Neuropsychological Theory. New York: Wiley.

Hilgetag, C. C., Theoret, H., \& Pascual-Leone, A. (2001). Enhanced visual spatial attention ipsilateral to rTMS-induced 'virtual lesions' of human parietal cortex. Nature Neuroscience, 4(9), 953-957.

Hoffman, R. E., Gueorguieva, R., Hawkins, K. A., Varanko, M., Boutros, N. N., Wu, Y. T., et al. (2005). Temporoparietal transcranial magnetic stimulation for auditory hallucinations: Safety, efficacy and moderators in a fifty patient sample. Biological Psychiatry, 58(2), 97-104.

Huang, Y. Z., Edwards, M. J., Rounis, E., Bhatia, K. P., \& Rothwell, J. C. (2005). Theta burst stimulation of the human motor cortex. Neuron, 45(2), 201-206.

Hummel, F. C., \& Cohen, L. G. (2006). Non-invasive brain stimulation: A new strategy to improve neurorehabilitation after stroke? Lancet Neurology, 5(8), 708-712.

Ilmoniemi, R. J., Virtanen, J., Ruohonen, J., Karhu, J., Aronen, H. J., Naatanen, R., et al. (1997). Neuronal responses to magnetic stimulation reveal cortical reactivity and connectivity. Neuroreport, 8(16), 3537-3540.

Kakuda, W., Abo, M., Kaito, N., Watanabe, M., \& Senoo, A. (2010). Functional MRI-based therapeutic rTMS strategy for aphasic stroke patients: A case series pilot study. International Journal of Neuroscience, 120(1), 60-66.

Kammer, T., \& Baumann, L. W. (2010). Phosphene thresholds evoked with single and double TMS pulses. Clinical Neurophysiology, 121(3), 376-379.

Kim, Y. H., Min, S. J., Ko, M. H., Park, J. W., Jang, S. H., \& Lee, P. K. (2005). Facilitating visuospatial attention for the contralateral hemifield by repetitive TMS on the posterior parietal cortex. NeuroscienceLetters, 382(3), 280-285.

Kirschen, M. P., Davis-Ratner, M. S., Jerde, T. E., Schraedley-Desmond, P., \& Desmond, J. E. (2006). Enhancement of phonological memory following transcranial magnetic stimulation (TMS). Behav Neurology, 17(3-4), 187-194.

Kleim, J. A., Bruneau, R., VandenBerg, P., MacDonald, E., Mulrooney, R., \& Pocock, D. (2003). Motor cortex stimulation enhances motor recovery and reduces peri-infarct dysfunction following ischemic insult. Neurology Research, 25(8), 789-793. 
Kleim, J. A., Chan, S., Pringle, E., Schallert, K., Procaccio, V., Jimenez, R., et al. (2006). BDNF val66met polymorphism is associated with modified experience-dependent plasticity in human motor cortex. Nature Neuroscience, 9(6), 735-737.

Klimesch, W., Sauseng, P., \& Gerloff, C. (2003). Enhancing Cognitive performance with repetitive transcranial magnetic stimulation at human individual alpha frequency. European Journal of Neuroscience, 17(5), 1129-1133.

Kobayashi, M., \& Pascual-Leone, A. (2003). Transcranial magnetic stimulation in neurology. Lancet Neurology, 2(3), 145-156.

Kohler, S., Paus, T., Buckner, R. L., \& Milner, B. (2004). Effects of left inferior prefrontal stimulation on episodic memory formation: A two-stage fMRI-rTMS study. Journal of Cognitive Neuroscience, 16(2), 178-188.

Komssi, S., \& Kahkonen, S. (2006). The novelty value of the combined use of electroencephalography and transcranial magnetic stimulation for neuroscience research. Brain Research Reviews, 52(1), 183-192.

Krings, T., Buchbinder, B. R., Butler, W. E., Chiappa, K. H., Jiang, H. J., Rosen, B. R., et al. (1997). Stereotactic transcranial magnetic stimulation: Correlation with direct electrical cortical stimulation. Neurosurgery, 41(6), 1319-1325, discussion 1325-1316.

Liebetanz, D., Nitsche, M. A., \& Paulus, W. (2003). Pharmacology of transcranial direct current stimulation: Missing effect of riluzole. Supplemental Clinical Neurophysiology, 56, 282-287.

Liepert, J., Bauder, H., Wolfgang, H. R., Miltner, W. H., Taub, E., \& Weiller, C. (2000). Treatment-induced cortical reorganization after stroke in humans. Stroke, 31(6), 1210-1216.

Liepert, J., Miltner, W. H., Bauder, H., Sommer, M., Dettmers, C., Taub, E., et al. (1998). Motor cortex plasticity during constraint-induced movement therapy in stroke patients. Neuroscience Letters, 250(1), 5-8.

Lu, B. (2003). BDNF and activity-dependent synaptic modulation. Learn Memory, 10(2), 86-98.

Luber, B., Kinnunen, L. H., Rakitin, B. C., Ellsasser, R., Stern, Y., \& Lisanby, S. H. (2007). Facilitation of performance in a working memory task with rTMS stimulation of the precuneus: frequency- and time-dependent effects. Brain Research, 1128(1), 120-129.

Maeda, F., Keenan, J. P., Tormos, J. M., Topka, H., \& Pascual-Leone, A. (2000). Modulation of corticospinal excitability by repetitive transcranial magnetic stimulation. Clinical Neurophysiology, 111(5), 800-805.

Mally, J., \& Stone, T. W. (2007). New advances in the rehabilitation of CNS diseases applying rTMS. Expert Reviews in Neurotherapy, 7(2), 165-177.

Manenti, R., Tettamanti, M., Cotelli, M., Miniussi, C., \& Cappa, S. F. (2010). The neural bases of word encoding and retrieval: A fMRI-guided transcranial magnetic stimulation study. Brain Topography, 22(4), 318-332.

Mariorenzi, R., Zarola, F., Caramia, M. D., Paradiso, C., \& Rossini, P. M. (1991). Non-invasive evaluation of central motor tract excitability changes following peripheral nerve stimulation in healthy humans. Electroencephalography and Clinical Neurophysiology, 81(2), 90-101.

Martin, P. I., Naeser, M. A., Theoret, H., Tormos, J. M., Nicholas, M., Kurland, J., et al. (2004). Transcranial magnetic stimulation as a complementary treatment for aphasia. Seminars in Speech Language, 25(2), 181-191.

McIntyre, C. C., Savasta, M., Walter, B. L., \& Vitek, J. L. (2004). How does deep brain stimulation work? Present understanding and future questions. Journal of Clinical Neurophysiology, 21(1), 40-50.

Meinzer, M., Flaisch, T., Breitenstein, C., Wienbruch, C., Elbert, T., \& Rockstroh, B. (2008). Functional re-recruitment of dysfunctional brain areas predicts language recovery in chronic aphasia. Neuroimage, 39(4), 2038-2046.

Miltner, W. H., Bauder, H., Sommer, M., Dettmers, C., \& Taub, E. (1999). Effects of constraintinduced movement therapy on patients with chronic motor deficits after stroke: A replication. Stroke, 30(3), 586-592. 
Miniussi, C., Cappa, S. F., Cohen, L. G., Floel, A., Fregni, F., Nitsche, M. A., et al. (2008). Efficacy of repetitive transcranial magnetic stimulation/transcranial direct current stimulation in cognitive neurorehabilitation. Brain Stimulation, 1(4), 326-336.

Miniussi, C., Ruzzoli, M., \& Walsh, V. (2010). The mechanism of transcranial magnetic stimulation in cognition. Cortex, 46(1), 128-130.

Miniussi, C., \& Thut, G. (2010). Combining TMS and EEG offers new prospects in cognitive neuroscience. Brain Topogr, 22(4), 249-256.

Mottaghy, F. M., Hungs, M., Brugmann, M., Sparing, R., Boroojerdi, B., Foltys, H., et al. (1999). Facilitation of picture naming after repetitive transcranial magnetic stimulation. Neurology, 53(8), 1806-1812.

Naeser, M. A., Martin, P. I., Lundgren, K., Klein, R., Kaplan, J., Treglia, E., et al. (2010). Improved language in a chronic nonfluent aphasia patient after treatment with CPAP and TMS. Cognitive and Behavioral Neurology, 23(1), 29-38.

Naeser, M. A., Martin, P. I., Nicholas, M., Baker, E. H., Seekins, H., Helm-Estabrooks, N., et al. (2005). Improved naming after TMS treatments in a chronic, global aphasia patient: Case report. Neurocase, 11(3), 182-193.

Naeser, M. A., Martin, P. I., Nicholas, M., Baker, E. H., Seekins, H., Kobayashi, M., et al. (2005). Improved picture naming in chronic aphasia after TMS to part of right Broca's area: An open-protocol study. Brain and Language, 93(1), 95-105.

Nakamura, H., Kitagawa, H., Kawaguchi, Y., \& Tsuji, H. (1996). Direct and indirect activation of human corticospinal neurons by transcranial magnetic and electrical stimulation. Neuroscience Letters, 210(1), 45-48.

Nakamura, H., Kitagawa, H., Kawaguchi, Y., \& Tsuji, H. (1997). Intracortical facilitation and inhibition after transcranial magnetic stimulation in conscious humans. Journal of Physiology, 498(Pt 3), 817-823.

Nardone, R., \& Tezzon, F. (2002). Inhibitory and excitatory circuits of cerebral cortex after ischaemic stroke: Prognostic value of the transcranial magnetic stimulation. Electromyography and Clinical Neurophysiology, 42(3), 131-136.

Nowak, D. A., Grefkes, C., Ameli, M., \& Fink, G. R. (2009). Interhemispheric competition after stroke: Brain stimulation to enhance recovery of function of the affected hand. Neurorehabilitation and Neural Repair, 23(7), 641-656.

Nudo, R. J., Wise, B. M., SiFuentes, F., \& Milliken, G. W. (1996). Neural substrates for the effects of rehabilitative training on motor recovery after ischemic infarct. Science, 272(5269), 1791-1794.

Oliveri, M., Rossini, P. M., Filippi, M. M., Traversa, R., Cicinelli, P., Palmieri, M. G., et al. (2000). Time-dependent activation of parieto-frontal networks for directing attention to tactile space. A study with paired transcranial magnetic stimulation pulses in right-braindamaged patients with extinction. Brain, 123(Pt 9), 1939-1947.

Oliveri, M., Rossini, P. M., Traversa, R., Cicinelli, P., Filippi, M. M., Pasqualetti, P., et al. (1999). Left frontal transcranial magnetic stimulation reduces contralesional extinction in patients with unilateral right brain damage. Brain, 122(Pt 9), 1731-1739.

Pasley, B. N., Allen, E. A., \& Freeman, R. D. (2009). State-dependent variability of neuronal responses to transcranial magnetic stimulation of the visual cortex. Neuron, 62(2), 291-303.

Paulus, W. (2011). Transcranial electrical stimulation (tES - tDCS; tRNS, tACS) methods. Neuropsychological Rehabilitation, 21(5), 602-617.

Pennisi, G., Rapisarda, G., Bella, R., Calabrese, V., Maertens De Noordhout, A., \& Delwaide, P. J. (1999). Absence of response to early transcranial magnetic stimulation in ischemic stroke patients: Prognostic value for hand motor recovery. Stroke, 30(12), 2666-2670.

Plautz, E. J., Barbay, S., Frost, S. B., Friel, K. M., Dancause, N., Zoubina, E. V., et al. (2003). Postinfarct cortical plasticity and behavioral recovery using concurrent cortical stimulation and rehabilitative training: A feasibility study in primates. Neurology Research, 25(8), 801-810. 
Post, A., Muller, M. B., Engelmann, M., \& Keck, M. E. (1999). Repetitive transcranial magnetic stimulation in rats: Evidence for a neuroprotective effect in vitro and in vivo. European Journal of Neuroscience, 11(9), 3247-3254.

Ridding, M. C., \& Rothwell, J. C. (2007). Is there a future for therapeutic use of transcranial magnetic stimulation? Nature Reviews Neuroscience, 8(7), 559-567.

Ridding, M. C., \& Ziemann, U. (2010). Determinants of the induction of cortical plasticity by non-invasive brain stimulation in healthy subjects. Journal of Physiology, 588( $\mathrm{Pt} 13)$, 2291-2304.

Rossi, S., Ferro, M., Cincotta, M., Ulivelli, M., Bartalini, S., Miniussi, C., et al. (2007). A real electro-magnetic placebo (REMP) device for sham transcranial magnetic stimulation (TMS). Clinical Neurophysiology, 118(3), 709-716.

Rossi, S., Hallett, M., Rossini, P. M., Pascual-Leone, A., \& Safety of T. M. S. C. G (2009). Safety, ethical considerations, and application guidelines for the use of transcranial magnetic stimulation in clinical practice and research. Clinical Neurophysiol, 120(12), 2008-2039.

Rossi, S., Rossini, P. M. (2004). TMS in cognitive plasticity and the potential for rehabilitation. Trends in Cognitive Science, 8(6), 273-279.

Rossini, P. M., Barker, A. T., Berardelli, A., Caramia, M. D., Caruso, G., Cracco, R. Q., et al. (1994). Non-invasive electrical and magnetic stimulation of the brain, spinal cord and roots: Basic principles and procedures for routine clinical application. Report of an IFCN committee. Electroencephalography and Clinical Neurophysiology, 91(2), 79-92.

Rossini, P. M., Calautti, C., Pauri, F., \& Baron, J. C. (2003). Post-stroke plastic reorganisation in the adult brain. Lancet Neurology, 2(8), 493-502.

Rossini, P. M., Caramia, M., \& Zarola, F. (1987). Central motor tract propagation in man: Studies with non-invasive, unifocal, scalp stimulation. Brain Research, 415(2), 211-225.

Rossini, P. M., Desiato, M. T., \& Caramia, M. D. (1992). Age-related changes of motor evoked potentials in healthy humans: Non-invasive evaluation of central and peripheral motor tracts excitability and conductivity. Brain Research, 593(1), 14-19.

Rossini, P. M., \& Rossi, S. (1998). Clinical applications of motor evoked potentials. Electroencephalography and Clinical Neurophysiology, 106(3), 180-194.

Rossini, P. M., \& Rossi, S. (2007). Transcranial magnetic stimulation: Diagnostic, therapeutic, and research potential. Neurology, 68(7), 484-488.

Rossini, P. M., Rossini, L., \& Ferreri, F. (2010). Brain-behavior relations: Transcranial magnetic stimulation. IEEE Engineering in Medicine and Biology Magazine, 29(1), 84-95.

Roth, Y., Zangen, A., \& Hallett, M. (2002). A coil design for transcranial magnetic stimulation of deep brain regions. Journal of Clinical Neurophysiology, 19(4), 361-370.

Rothwell, J. C., Day, B. L., Thompson, P. D., Dick, J. P., \& Marsden, C. D. (1987). Some experiences of techniques for stimulation of the human cerebral motor cortex through the scalp. Neurosurgery, 20(1), 156-163.

Ruzzoli, M., Marzi, C. A., \& Miniussi, C. (2010). The neural mechanisms of the effects of transcranial magnetic stimulation on perception. Journal of Neurophysiology, 103(6), 2982-2989.

Sack, A. T., \& Linden, D. E. (2003). Combining transcranial magnetic stimulation and functional imaging in Cognitive brain research: possibilities and limitations. Brain Research Reviews, 43(1), 41-56.

Sack, A. T., Kadosh, R. C., Schuhmann, T., Moerel, M., Walsh, V., \& Goebel, R. (2009). Optimizing functional accuracy of TMS in cognitive studies: A comparison of methods. Journal of Cognitive Neuroscience, 21, 207-221.

Sandrini, M., Umilta, C., \& Rusconi, E. (2011). The use of transcranial magnetic stimulation in Cognitive neuroscience: A new synthesis of methodological issues. Neuroscience and Biobehavioral Reviews, 5(3), 516-536. 
Schabitz, W. R., Berger, C., Kollmar, R., Seitz, M., Tanay, E., Kiessling, M., et al. (2004). Effect of brain-derived neurotrophic factor treatment and forced arm use on functional motor recovery after small cortical ischemia. Stroke, 35(4), 992-997.

Selimbeyoglu, A., \& Parvizi, J. (2010). Electrical stimulation of the human brain: Perceptual and behavioral phenomena reported in the old and new literature. Frontiers in Human Neuroscience, 4, 46.

Sgro, J. A., Ghatak, N. R., Stanton, P. C., Emerson, R. G., \& Blair, R. (1991). Repetitive high magnetic field stimulation: The effect upon rat brain. Electroencephalography and Clinical Neurophysiology Supplement, 43, 180-185.

Shindo, K., Sugiyama, K., Huabao, L., Nishijima, K., Kondo, T., \& Izumi, S. (2006). Long-term effect of low-frequency repetitive transcranial magnetic stimulation over the unaffected posterior parietal cortex in patients with unilateral spatial neglect. Journal of Rehabilitation Medicine, 38(1), 65-67.

Silvanto, J., Cowey, A., Lavie, N., \& Walsh, V. (2005). Striate cortex (V1) activity gates awareness of motion. Nature Neuroscience, 8(2), 143-144.

Silvanto, J., Muggleton, N. G., Cowey, A., \& Walsh, V. (2007). Neural activation state determines behavioral susceptibility to modified theta burst transcranial magnetic stimulation. European Journal of Neuroscience, 26(2), 523-528.

Silvanto, J., Muggleton, N., \& Walsh, V. (2008). State-dependency in brain stimulation studies of perception and cognition. Trends in Cognitive Science, 12(12), 447-454.

Sommer, M., Tergau, F., Wischer, S., \& Paulus, W. (2001). Paired-pulse repetitive transcranial magnetic stimulation of the human motor cortex. Experimental Brain Research, 139(4), 465-472.

Sparing, R., Mottaghy, F. M., Hungs, M., Brugmann, M., Foltys, H., Huber, W., et al. (2001). Repetitive transcranial magnetic stimulation effects on language function depend on the stimulation parameters. Journal of Clinical Neurophysiology, 18(4), 326-330.

Stefan, K., Kunesch, E., Benecke, R., Cohen, L. G., \& Classen, J. (2002). Mechanisms of enhancement of human motor cortex excitability induced by interventional paired associative stimulation. Journal of Physiology, 543(Pt 2), 699-708.

Takeuchi, N., Tada, T., Toshima, M., Matsuo, Y., \& Ikoma, K. (2009). Repetitive transcranial magnetic stimulation over bilateral hemispheres enhances motor function and training effect of paretic hand in patients after stroke. Journal of Rehabilitation Medicine, 41(13), 1049-1054.

Talelli, P., \& Rothwell, J. (2006). Does brain stimulation after stroke have a future? Current Opinion in Neurology, 19(6), 543-550.

Thickbroom, G. W. (2007). Transcranial magnetic stimulation and synaptic plasticity: Experimental framework and human models. Expimental Brain Research, 180(4), 583-593.

Thickbroom, G. W., Sammut, R., \& Mastaglia, F. L. (1998). Magnetic stimulation mapping of motor cortex: Factors contributing to map area. Electroencephalography and Clinical Neurophysiology, 109(2), 79-84.

Thielscher, A., \& Kammer, T. (2004). Electric field properties of two commercial figure-8 coils in TMS: Calculation of focality and efficiency. Clinical Neurophysiology, 115(7), 1697-1708.

Thut, G., \& Miniussi, C. (2009). New insights into rhythmic brain activity from TMS-EEG studies. Trends in Cognitive Science, 13(4), 182-189.

Thut, G., \& Pascual Leone, A. (2010). A review of combined TMS-EEG studies to characterize lasting effects of repetitive TMS and assess their usefulness in cognitive and clinical neuroscience. Brain Topography, 22, 4.

Traversa, R., Cicinelli, P., Bassi, A., Rossini, P. M., \& Bernardi, G. (1997). Mapping of motor cortical reorganization after stroke. A brain stimulation study with focal magnetic pulses. Stroke, 28(1), 110-117. 
Vallar, G., \& Bolognini, N. (2011). Behavioural facilitation following brain stimulation: Implications for neurorehabilitation. Neuropsychological Rehabilitation, 21(5), 618-649.

Vallesi, A., Shallice, T., \& Walsh, V. (2007). Role of the prefrontal cortex in the foreperiod effect: TMS evidence for dual mechanisms in temporal preparation. Cerebral Cortex, 17(2), 466-474.

Virtanen, J., Ruohonen, J., Naatanen, R., \& Ilmoniemi, R. J. (1999). Instrumentation for the measurement of electric brain responses to transcranial magnetic stimulation. Medical and Biological Engineering and Computing, 37(3), 322-326.

Walsh, V., \& Pascual-Leone, A. (2003). Transcranial Magnetic Stimulation: A Neurochronometrics of Mind. Cambridge, MA: Mit Press.

Wang, H., Wang, X., \& Scheich, H. (1996). LTD and LTP induced by transcranial magnetic stimulation in auditory cortex. Neuroreport, 7(2), 521-525.

Ward, N. S., \& Cohen, L. G. (2004). Mechanisms underlying recovery of motor function after stroke. Archives in Neurology, 61(12), 1844-1848.

Wassermann, E. M., Epstein, C., Ziemann, U., Walsh, V., Paus, T., \& Lisanby, S. (2008). Handbook of Transcranial Stimulation. Oxford, UK: Oxford University Press.

Wassermann, E. M., \& Lisanby, S. H. (2001). Therapeutic application of repetitive transcranial magnetic stimulation: A review. Clinical Neurophysiology, 112(8), 1367-1377.

Waterston, M. L., \& Pack, C. C. (2010). Improved discrimination of visual stimuli following repetitive transcranial magnetic stimulation. Public Library of Science: One, 5(4), e10354.

Wittenberg, G. F., Chen, R., Ishii, K., Bushara, K. O., Eckloff, S., Croarkin, E., et al. (2003). Constraint-induced therapy in stroke: Magnetic-stimulation motor maps and cerebral activation. Neurorehabilitation and Neural Repair, 17(1), 48-57.

Zangen, A., Roth, Y., Voller, B., \& Hallett, M. (2005). Transcranial magnetic stimulation of deep brain regions: Evidence for efficacy of the H-coil. Clinical Neurophysiology, 116(4), 775-779.

Zhang, X., Mei, Y., Liu, C., \& Yu, S. (2007). Effect of transcranial magnetic stimulation on the expression of c-Fos and brain-derived neurotrophic factor of the cerebral cortex in rats with cerebral infarct. Journal of Huazhong University Science, Technology and Medical Science, 27(4), 415-418.

Ziemann, U., Chen, R., Cohen, L. G., \& Hallett, M. (1998). Dextromethorphan decreases the excitability of the human motor cortex. Neurology, 51(5), 1320-1324.

Ziemann, U., Hallett, M., \& Cohen, L. G. (1998). Mechanisms of deafferentation-induced plasticity in human motor cortex. Journal of Neuroscience, 18(17), 7000-7007. 